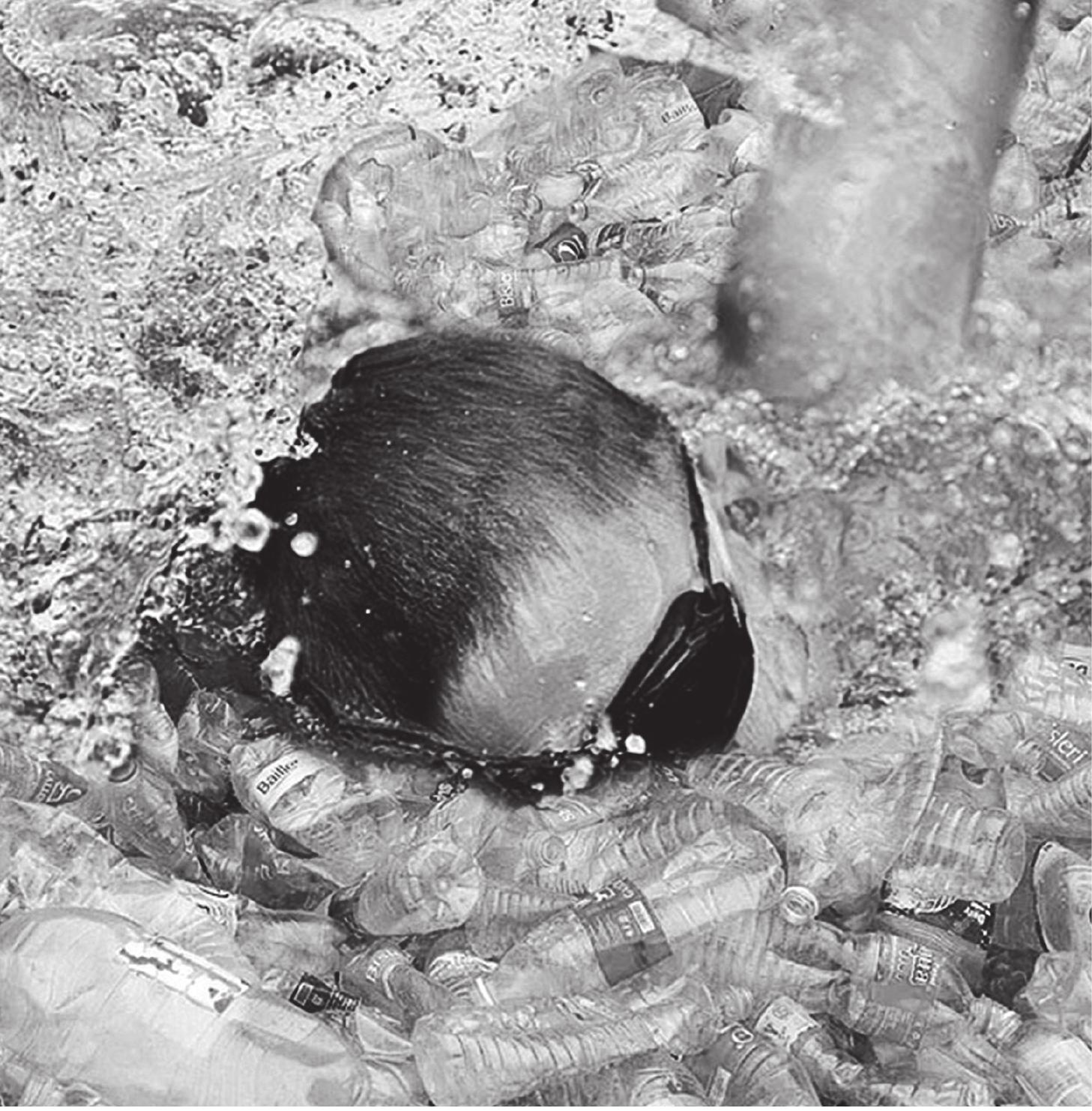

AIBR

Revista de Antropología

Iberoamericana

www.aibr.org

Volumen 14

Número 1

Enero - Abril 2019

Pp. 11 - 27

Madrid: Antropólogos Iberoamericanos en Red. ISSN: 1695-9752 E-ISSN: 1578-9705
Corporalidades permeables: Intersecciones entre medio ambiente y salud

\section{Introducción al monográfico}

Arantza Begueria

Observatorio de la Alimentación, Universitat de Barcelona

\section{Eva Zafra-Aparici}

Universitat Rovira i Virgili

Recibido: 08.11.2018

Aceptado: 15.11 .2018

DOI: $10.11156 / a i b r .140102$ 


\section{RESUMEN}

A partir de la segunda mitad del siglo XX, el desarrollo tecnológico en diversos campos industriales ha provocado que los seres humanos convivan, en mayor o menor medida, con diversos compuestos químicos sintéticos presentes en la atmósfera, el agua, la tierra y los alimentos. Estos compuestos han afectado, afectan y afectarán la salud de diversos grupos de población en distintas formas. El presente trabajo propone una visión del cuerpo contemporáneo pensado a partir de las múltiples relaciones entre medio ambiente y salud. En primer lugar, se realiza un somero recorrido por la literatura antropológica en torno a estos temas, sus planteamientos y preocupaciones, en el ámbito de la salud, el riesgo, la cultura, las instituciones políticas, la ciencia, y, especialmente, la alimentación. A continuación, se plantea el concepto de «corporalidades permeables» para designar el momento en que la toxicidad proveniente del medio ambiente se incorpora en los cuerpos humanos y los convierte en cuerpos permeables, relacionales y abiertos al mundo que los rodea. Finalmente, se presentan los trabajos que forman parte de este monográfico. Aquí se incluyen tres trabajos etnográficos en zonas con alta contaminación en Ghana, Argentina y Vietnam. Completan el monográfico otras tres contribuciones que tienen como eje el riesgo en la alimentación y que tratan sobre los alimentos funcionales en Argentina, los alimentos y su toxicidad en embarazadas y lactantes en España, y sobre las clasificaciones y elecciones alimentarias en la sociedad española.

\section{PALABRAS CLAVE}

Toxicidad, medio ambiente, cuerpo, riesgo, alimentación.

\section{PERMEABLE CORPORALITIES: INTERSECTIONS BETWEEN THE ENVIRONMENT AND HEALTH. PRESENTATION TO THE MONOGRAPH}

\section{ABSTRACT}

From the second half of the twentieth century, due to technological developments in various industrial fields, humans have to coexist, to a greater or lesser extent, with various synthetic chemical compounds that can be found in air, water, land, and food. These compounds have affected, affect, and will continue to affect the health of many population groups in different ways. This Special Issue presents a space to think about the contemporary body through the multiple relationships between environment and health. First, this article introduces some anthropological literature around these issues, its approaches and concerns, in the field of health, risk, culture, politics, science, and especially food. Next, the concept of "permeable corporalities" is raised to designate the moment in which the toxicity coming from the environment impregnates human bodies and turns them into permeable, relational bodies, open to the world that surrounds them. Finally, the works that are part of this Special Issue are presented. This includes three ethnographic studies in areas with high levels of pollution in Ghana, Argentina, and Vietnam. The Special Issue also presents three other contributions that focus on food risk and which deal with functional foods in Argentina, food toxicity in pregnant and breastfeeding women in Spain, and on food classifications and choices in Spanish society.

\section{KEY WORDS}

Toxicity, environment, body, risk, food. 
Han pasado más de 30 años desde que Ulrich Beck bautizó la sociedad contemporánea como "la sociedad del riesgo» (Beck, 1986). Pese a que ya existían estudios anteriores sobre riesgo ambiental (Douglas y Wildavsky, 1982) y aunque se puede debatir sobre algunos de los aspectos antropológicos del trabajo de Beck (Jensen y Blok, 2008), es evidente que la expresión "sociedad del riesgo" ha servido para pensar la sociedad contemporánea, sus retos, sus contradicciones y su futuro. Tal y como este autor vaticinó, algunos de los riesgos contemporáneos más sobresalientes en las sociedades del siglo XXI se sitúan en el ámbito medioambiental y a nivel de salud local y global.

A partir de algunos desarrollos tecnológicos en la producción agrícola, industrial, química, textil y alimentaria de las últimas décadas, puede decirse que actualmente vivimos rodeados de compuestos químicos sintéticos presentes en la atmósfera, el agua, la tierra y los alimentos. Estos compuestos llegan al ser humano a través de la respiración, la alimentación y el contacto cutáneo. Pueden suponer riesgos con efectos a gran escala que son difíciles de prever y de tratar, y que pueden constituir una amenaza silenciosa, pero violentamente real.

El medio ambiente ha devenido una preocupación global. El cambio climático, la destrucción de la biodiversidad, la polución en grandes ciudades, la acumulación de despojos electrónicos y químicos en países del Tercer Mundo, las grandes islas de residuos plásticos en los océanos, la basura espacial que orbita la Tierra, así como la utilización de pesticidas u otros productos químicos en la cadena alimentaria, son, entre muchos otros, algunos ejemplos de riesgos cuyos efectos son a la vez locales y globales, presentes y futuros.

La idea de este monográfico surgió a partir del panel del mismo nombre que se celebró en el II Congreso Internacional de Antropología AIBR (Barcelona, 2016). Aquí se presentan trabajos que analizan antropológicamente las intersecciones entre medio ambiente y salud bajo una perspectiva centrada en el cuerpo como lugar donde observar las implicaciones individuales, sociales y políticas de estos fenómenos. Este monográfico propone el término de «corporalidades permeables» para referirse a los cuerpos humanos como cuerpos expuestos a un medio ambiente cambiante y alterado y a los posibles efectos de este sobre la salud humana.

\section{Riesgo, medio ambiente y salud humana}

La antropología se ha ocupado del medio ambiente desde su nacimiento como disciplina, pues el hábitat en el que viven las comunidades analizadas por los antropólogos es parte esencial de su vida social. La ecología 
cultural, la ecología política, la antropología de la salud, la antropología del riesgo o los estudios de ciencia y tecnología, entre otros, son disciplinas que se han ocupado específicamente del análisis del medio ambiente y su relación con todas las esferas de la vida social (Haenn y Wilk, 2006).

Este monográfico se centra específicamente en la relación entre el medio ambiente, la salud y el cuerpo humano. Desde la clásica Primavera Silenciosa (Carson, 1964), que advertía de los efectos de los pesticidas sobre la salud humana, y que inspiró en gran parte a los movimientos ecologistas del siglo XX, una vasta producción académica se ha dedicado a analizar esta relación, casi nunca exenta de polémica. En la antropología de la salud y en disciplinas afines, uno de los temas recurrentes ha sido el análisis de las formas en que se perciben y conceptualizan los riesgos para la salud en distintas sociedades o circunstancias, así como en las maneras de afrontarlos en la vida cotidiana (Harthorn, 2003; Hunt, Tinoco, Schwartz y Halperin, 1999; Larrea-Killinger, Muñoz, Mascaró, Zafra y Porta, 2017; Palis, Flor, Warburton y Hossain, 2006; Quandt, Arcury, Austin y Saavedra, 1998; Snipes, Thompson, O'Connor, Shell-Duncan, King, Herrera y Navarro, 2009) y en función de los distintos discursos —científicos, históricos, medioambientales, de la salud, entre otros-que conforman los saberes sociales y comunitarios sobre los riesgos (Altman, Morello-Frosch, Brody, Rudel, Brown y Averick, 2008). Estos trabajos muestran las formas en que los factores culturales, sociales, económicos y personales pueden hacer que se perciban distintos grados de toxicidad en determinadas sustancias, e incluso diferentes niveles de riesgo percibido dependiendo de las situaciones sociales. A este respecto, por ejemplo, Erickson (2007) o Soares de Freitas y sus colegas (Soares de Freitas, de Souza Minayo, Lopes Pena y Miranda dos Santos, 2012) proponen usar el concepto de «modelos explicativos» de Kleinman (1980) para entender los distintos gradientes de percepción del riesgo medioambiental según los diversos contextos sociales. De hecho, ya desde los años 70 del siglo XX, los trabajos de la antropóloga Mary Douglas sugerían que los factores simbólicoculturales se encontraban en la base de la percepción de cualquier tipo de riesgo (Douglas, 1994; Douglas y Wildavsky, 1982).

Ya se trate de pesticidas, radiación nuclear, contaminación industrial o cualquier otro tipo de peligro, la bibliografía de la antropología médica y disciplinas afines examinan, en última instancia, las creaciones de sentido. Así, se trata de dilucidar cuáles son los factores, los elementos sociales y culturales que proporcionan sentido a unas u otras explicaciones de los efectos del medio ambiente sobre la salud humana o, más concretamente, de las imbricaciones entre ambos. En este engranaje, varios campos juegan papeles entrelazados y superpuestos. Por un lado, el campo político ha 
sido analizado como uno de los actores relevantes en este contexto. Firmemente ligado a este, el campo de la producción científica ha tenido un papel destacado en los análisis, en especial por las controversias e incertidumbres creadas por la ciencia y los actores sociales implicados en ella (Boudia y Jas, 2014; Checker, 2007). En esta línea, diversos trabajos han analizado cómo se construyen los «hechos científicos» y la consecuente legitimación o deslegitimación política de determinados padecimientos (Petryna, 2013; Phillips, 2010), así como su relación con los llamados «conocimientos no expertos» (Wynne, 1996). Otro de los actores principales del teatro de los daños medioambientales son las industrias que contaminan el medio ambiente, también frecuentemente en comunión con los gobiernos locales (Sicotte, 2009). Finalmente, el papel de los medios de comunicación como creadores de sentidos sociales ha despertado también el interés de las ciencias sociales (Begueria, Larrea, Muñoz, Zafra, Mascaró-Pons y Porta, 2014; Brown, Zavestoski, McCormick, Mandelbaum y Luebke, 2001).

A lo largo de los trabajos citados y de otros que no alcanzamos a incluir aquí, el binomio desigualdad-incertidumbre crea un patrón que se repite a través de distintas sociedades y momentos históricos. Los trabajos de Auyero y Swintun (2008), por ejemplo, reflexionan sobre las condiciones políticosociales de creación de dudas e incertidumbre entre la población de un barrio de chabolas argentino respecto a la situación de sufrimiento ambiental en la que viven. Con el mismo planteamiento, Lora-Wainwright (2013) analiza cómo se generan la evidencia y la incertidumbre en dos poblaciones chinas, ambas afectadas por altos niveles de polución. Vinculada a esta cuestión, la desigualdad social aparece de forma recurrente, pues la creación de incertidumbre social afecta, en muchos casos, a sectores social y económicamente precarizados de la población (Auyero y Swistun, 2008; Harthorn, 2003; Singer, 2011). En este sentido, algunos trabajos se centran en los elementos en torno a los cuales se estructura la reivindicación de legitimidad de aquellos ciudadanos afectados por problemas de salud provenientes del medioambiente (Phillips, 2010).

Entre las inseguridades medioambientales, merece especial atención la cuestión de la alimentación, pues es este uno de los lugares privilegiados donde convergen las ideas de riesgo ambiental, salud y cuerpo. Las numerosas alarmas alimentarias de las últimas décadas en las sociedades occidentales, junto con el aumento de sustancias químicas utilizadas en la producción agrícola e industrial, el incremento continuo de la oferta y la demanda de productos alimentarios diversos, y las distintas formas de producir, comer y consumir, están comportando cambios en las percepciones sociales sobre el riesgo alimentario y su relación con el medio 
ambiente. Al respecto, numerosos estudios socioantropológicos (Apfelbaum, 1998; Bredahl, 1999; Butz, Needs, Baron, Bayer, Geisel, Gupta, Oltersdorf et al., 2003; Espeitx y Cáceres, 2012; Fischler, 2002; Guidonet, 2010; Latouche, Rainelli y Vermersch, 1998; Zafra Aparici, Muñoz García y Larrea-Killinger, 2016) se han centrado en el análisis del riesgo en los alimentos, la cadena alimentaria industrializada y sus aplicaciones tecnológicas, focalizando el análisis, mayoritariamente, en los aspectos de la organización económica y política de nuestra sociedad actual.

En este sentido, el presente monográfico pretende expandir los planteamientos hasta aquí resumidos para centrar el debate en el cuerpo humano como arena de investigación antropológica. Este trabajo responde no solamente al relativo vacío existente en la literatura en español, sino también a una preocupación social emergente por las consecuencias encarnadas y corporales de ciertas prácticas gubernamentales, industriales y científicas en las sociedades globales.

\section{Corporalidades permeables}

La cita de Foucault según la cual el cuerpo es un campo de batalla donde se libran combates filosóficos, morales, científicos, culturales y sociales ha inspirado múltiples trabajos académicos y no académicos y nos resulta también especialmente sugerente para el tema que aquí se trata (LarreaKillinger, Muñoz y Mascaró, 2017). El cuerpo, los cuerpos de los ciudadanos, son idiomas privilegiados en los que se expresa la sociedad en su complejidad y están inevitablemente sujetos a sus contextos económicos, su situación política, su lugar culturalmente establecido y también, cómo no, a las formas en que esos contextos se expresan en el medio ambiente en el que viven, beben, respiran y comen. El momento en que ese medio ambiente se convierte en un riesgo o un peligro para los cuerpos, para su salud y su vida, es el lugar en que este monográfico se sitúa.

Tal y como pusieron de relieve las epidemiólogas Kavanagh y Broom (1998), el riesgo ha sido estudiado desde las ciencias sociales a partir de dos categorías aparentemente opuestas: la del «medio ambiente» y la de los «estilos de vida». El primero, un tipo de peligro entendido como externo al individuo y de tipo colectivo, sitúa la raíz del peligro en el sistema social, político o económico. El segundo se emplazaría en culpabilizar a las víctimas por sus factores de conducta individuales que supuestamente conllevarían un riesgo para la salud, omitiendo así los imperativos sociales, económicos y políticos que se hallan tras el concepto de «estilos de vida». En este sentido, e introduciendo el cuerpo humano como eje del 
análisis, estas autoras proponen la noción de embodied risk, un tipo riesgo que está in-corporado, corporalizado, pues no es totalmente medioambiental ni solamente individual, pero es ambas cosas a la vez, porque el cuerpo y el ambiente son inseparables. El cuerpo se entiende a la vez como un receptáculo de aquellos riesgos que provienen de fuera de este y como un sujeto activo en su construcción social.

La revista Culture, Theory and Critique ha publicado recientemente un monográfico dedicado al invisible harm, el daño invisible, un término utilizado para capturar los amplios efectos de una toxicidad ambiental en aumento en contextos específicos del capitalismo tardío (Goldstein, 2017). Esta toxicidad se caracteriza, precisamente, por ser invisible y poder solamente medirse mediante dispositivos tecnológicos. Nuestro monográfico quisiera situarse en diálogo con este concepto de «daño invisible», para ubicar este daño presente o futuro, precisamente, en los cuerpos humanos. En este sentido, la noción de embodied risk (Kavanagh y Broom, 1998) resulta especialmente interesante para el análisis que aquí se propone. Además, está relacionada con aquella de «contaminación interior»o «contaminación interna» (Porta, Puigdomènech y Ballester Díez, 2009), pues ambas presuponen que la toxicidad proveniente del medio ambiente se ha introducido en los cuerpos humanos y forma ya parte intrínseca de los mismos. Y, aunque la toxicidad en sí misma sea invisible en el medio ambiente, sus consecuencias son, o serán en el futuro, muy evidentes, tanto en la corporalidad física como en la psíquica y social de las personas. Asimismo, el concepto de «cuerpos tóxicos» (Larrea-Killinger, Muñoz y Mascaró, 2017; Larrea-Killinger et al., 2017) da cuenta de esa centralidad de la toxicidad contemporánea en los cuerpos humanos y de la necesidad de analizar las prácticas y discursos socioculturales de y desde la experiencia corporal de la contaminación interna.

Otra publicación reciente en la literatura anglosajona es el número de noviembre de 2017 de la revista Cultural Anthropology que, bajo la consigna de chemo-ethnographies -etnografías químicas- (Shapiro y Kirksey, 2017), recoge, entre otros, un artículo de Michelle Murphy sobre lo que ella denomina alter-life, es decir, la vida alterada por los compuestos químicos persistentes y, por ende, por el sistema que los crea, desarrolla, reproduce y legitima. Esta autora ha trabajado sobre diversos casos de contaminación química y ha acuñado términos como "regímenes de vida químicos», o alter-life (Murphy, 2008 y 2017a), dos conceptos que dan cuenta de la absoluta interrelación entre los humanos y su entorno, tanto a nivel molecular como social y político, y que tienen muy en cuenta las desigualdades e injusticias que se dan en entornos donde las problemáticas medioambientales han devenido acuciantes. 
De la misma forma, este monográfico se plantea a partir de la necesidad de analizar el riesgo medioambiental encarnado desde perspectivas que tengan en cuenta su multifactorialidad de forma relacional. Cada vez más, el cuerpo —individual, social y político (Scheper-Hughes y Lock, 1987) — va ocupando el centro de la producción del discurso y las prácticas alrededor del riesgo, el medio ambiente y la alimentación, de manera que las percepciones y las prácticas sobre el «cuerpo contaminado» no solamente se construyen conforme la visión biomédica de un cuerpo físico, químico o biológico, cuyo interior ha sido contaminado, sino también en función de la corporalidad individual, social y política que lo articula (Larrea-Killinger, Muñoz y Mascaró, 2017; Muñoz, Larrea, Zafra y Begueria, 2014; Zafra Aparici, Muñoz García y Larrea-Killinger, 2016). Es de especial importancia notar esta cuestión pues, tal y como nos recuerda Michelle Murphy (2017a y 2017b), si solamente tenemos en cuenta los cuerpos individuales y sus marcadores biológicos de exposición a sustancias tóxicas, podemos caer en la trampa de olvidar sus múltiples dimensiones sociales y políticas, así como contribuir a la patologización de comunidades que ya viven bajo la "violencia química», en condiciones hostiles o de desigualdad social.

En este sentido, partimos de referentes clásicos anglosajones como los de la antropóloga Mary Douglas, que afirmaba que el análisis del riesgo debería considerar primordialmente los factores simbólicoculturales de los cuerpos y las formas de organización social (Douglas y Wildavsky, 1982). Siguiendo esta línea, la socióloga Deborah Lupton ha estado trabajando durante años en la cuestión corporal en el estudio socioantropológico del riesgo, donde el cuerpo se plantea como construido y experimentado a través de discursos, conocimientos y estrategias sobre riesgo. La autora ha explorado las dimensiones de riesgo en diversos niveles de significado socioestructural, cultural y simbólico, y en cómo los cuerpos viven esta experiencia (Lupton, 1999a y 2013; Tulloch y Lupton, 2003).

Los trabajos citados ponen en evidencia que el cuerpo puede constituir el centro a partir del cual se construyan los discursos sobre el riesgo contemporáneo y sus múltiples batallas ideológicas. En esas batallas, el cuerpo no solamente es un objeto pasivo, al modo de los cuerpos dóciles de Foucault (2009), al que van a parar y acumularse sustancias potencialmente nocivas, sino también un sujeto activo del proceso de construcción de discursos y saberes y con capacidad individual, social y política de transformación hacia un mundo mejor y más seguro (Zafra Aparici, Muñoz García y Larrea-Killinger, 2016). Este doble rol no implica olvidar, además, el hecho de que no todos los sujetos se encuentran equitativamen- 
te situados en posiciones sociales, y de poder para ejercer estas capacidades de igual manera (Murphy, 2017a).

Recogiendo estos planteamientos, este monográfico propone el término de "corporalidades permeables» para referirse al cuerpo del sujeto contemporáneo, radicalmente relacional, cuyo riesgo encarnado se encuentra atravesado por discursos, prácticas, conceptos, artefactos y sustancias que lo colocan en el foco de atención de algunos trabajos que aquí se presentan.

A lo largo de la historia, los cuerpos humanos se han percibido como abiertos o cerrados en función de su relación con la otredad, es decir, con el mundo que les rodeaba. Ejemplo de ello sería el cuerpo medieval, abierto a los demás, al universo y a la comunidad, en oposición al cuerpo civilizado, por ejemplo, individualista, dualista y separado del «otro» ( Elias, 1989; Le Breton, 2002). En este monográfico proponemos una visión del cuerpo contemporáneo como irremediablemente abierto al mundo que le rodea, a partir de pensarlo en relación con la toxicidad medioambiental. La idea del tóxico que habita ya dentro del cuerpo humano diluye la frontera clásica entre el individuo y la sociedad, pues aquello que proviene del medio ambiente ha atravesado ya el perímetro del individuo y se sitúa tanto en su interior como en su exterior. La frontera simbólica entre interior-exterior ha cesado de existir, y las personas somos ya parte del medio ambiente químico a nivel íntimo y molecular. Las clásicas dualidades individuo-sociedad, o naturaleza-cultura, con las que los antropólogos han bregado durante años, quedan superadas por esa noción de contaminación que atraviesa las fronteras de los cuerpos, ya sea por vía respiratoria, cutánea o alimentaria. La fractura de esa frontera convierte los cuerpos contemporáneos en cuerpos permeables, expuestos y porosos, cuerpos atravesados por el medio ambiente en que habitan y sus contextos sociales y culturales particulares (Begueria, 2016; Larrea-Killinger, Muñoz y Mascaró, 2017; Mascaró Pons, 2013).

Conviene subrayar una vez más que la noción de cuerpos permeables no implica que los cuerpos sean meramente vulnerables o pasivos, sino que más bien enfatiza su radical relacionalidad, su contacto íntimo y constante con el mundo a su alrededor, humano y no-humano; y, con esto, su capacidad activa tanto para ser afectado (Latour, 2004) como para actuar en consecuencia. Es precisamente esa capacidad relacional la que confiere a los cuerpos, a las personas, su oportunidad de conformarse en comunidades que articulan sus derechos y su capacidad política de ser sujetos activos (Petryna, 2013), a la vez vulnerables y porosos, abiertos al mundo que los rodea, aunque sea este un mundo rebosante de compuestos químicos y seamos, todos nosotros, cuerpos irremediablemente permeables. 


\section{Artículos y contribuciones}

Los artículos que se recogen en este monográfico presentan distintas visiones de riesgos medioambientales en lugares del mundo como Vietnam, Ghana, Argentina o España. Los cuerpos presentes en estos lugares se viven y se conceptualizan de formas histórica y socialmente situadas, tal y como se presenta en cada uno de los textos.

El artículo de Takeshi Uesugi propone entender el riesgo medioambiental y sus percepciones sociales desde una perspectiva dialógica y compleja, que tiene en cuenta discursos científicos, narrativas históricas, itinerarios corporales y factores culturales y políticos. Su etnografía se realizó en el valle de A Luoi, en el centro de Vietnam, donde en los años 60 y 70 del siglo XX, durante la Segunda Guerra de Indochina, el ejército americano roció la zona con un herbicida conocido como Agente Naranja, que contaminó tierras, ríos y personas, y que continúa causando, a día de hoy, muertes, enfermedades y malformaciones corporales.

En su artículo, Uesugi analiza los significados sociales y corporales asociados al Agente Naranja de los habitantes del valle de A Luoi a partir del momento en que varios estudios científicos verifican la presencia de este compuesto químico en el valle. El autor no se centra específicamente en la forma en que el conocimiento sobre esta sustancia tóxica forma una colectividad política a través de redefinir síntomas que anteriormente no se identificaban con el Agente Naranja, sino que se focaliza más bien en explicar las maneras de entender subjetiva y afectivamente las situaciones de riesgo de los habitantes de A Luoi. El autor señala la incertidumbre — pasada, presente y futura- como uno de los elementos clave para comprender las ideas de riesgo de la población, que irrumpen en momentos concretos de su vida cotidiana, en sus encuentros con otros seres humanos, afectados no, o con diversas materialidades tóxicas. Esas percepciones son a la vez culturales, históricas, encarnadas y afectivas.

El artículo de Peter Little se basa en un trabajo etnográfico en la zona de Agbogbloshie, en la República de Ghana, que está considerado uno de los lugares más contaminados del mundo por la presencia de grandes cantidades de basura electrónica proveniente del Norte global. Esa basura se recicla en Ghana, tanto en plantas procesadoras formales como a través de un gran mercado laboral informal de reciclaje al aire libre altamente contaminante, donde se aúnan la explotación laboral, el trabajo migrante, la falta de medidas protectoras y la escasa atención pública a la salud de estos trabajadores. El autor se centra en las narrativas corporales de uno de los trabajadores de reciclaje de esta región y su relación con las sustancias tóxicas que impregnan la tierra, el aire, el agua y los cuerpos. 
A partir de ahí, traza un recorrido por la política poscolonial de corporalidades disciplinadas, de desigualdad social y de contaminación, en un contexto racializado, para proponer el concepto de "corporalidades tóxicas poscoloniales", un concepto que une tierras, cuerpos, economías y tóxicos, para demostrar la interrelación entre estos elementos. Además, el autor destaca el papel de las ONG en esta zona que, mediante intervenciones supuestamente ecológicas, reproducen todavía más la situación vulnerable de los trabajadores migrantes y su culpabilización por su estado de salud.

Resulta especialmente interesante la relación explícita que propone este autor entre las políticas poscoloniales y capitalistas globales con la toxicidad encarnada en los cuerpos de aquellas comunidades que viven en situaciones hostiles, de precariedad económica, política y social.

De la misma forma, el artículo de Marcelo Sarlingo propone un recorrido histórico-antropológico por el Partido de Olavarría, en Argentina, y las condiciones sociopolíticas que han permitido, durante el último siglo, convertir la región en un área fuertemente contaminada por diversas industrias y con numerosos casos de enfermedades graves entre sus habitantes, entre otros efectos ambientales severos. El autor analiza este fenómeno, que se da gracias a una retroalimentación entre políticas industriales agresivas en el área de la minería y la gestión de residuos tóxicos, una tolerancia política desde las estructuras formales de poder, un $\mathrm{I}+\mathrm{D}$ al servicio de la industria y un ocultamiento sistemático por parte de los medios de comunicación.

Este silenciamiento de los fenómenos de contaminación va acompañado de representaciones simbólicas e ideológicas colectivas que han potenciado la inacción ciudadana y su expresión en los cuerpos de sus víctimas, en una suerte de naturalización del neoliberalismo en sus propios cuerpos, al que el autor se refiere como "hegemonías naturales» $\mathrm{O}$ "habitualidad tóxica», un fenómeno que también se observa en los casos de Ghana y Vietnam.

Es interesante advertir que las etnografías que se presentan en este monográfico no tratan de colectividades políticamente conformadas en torno a fenómenos tóxicos, como otras etnografías citadas anteriormente (Petryna, 2013), sino de comunidades en situaciones de precariedad y relativo desconocimiento de los factores ambientales que afectan directamente a sus vidas. En este sentido, sería significativo analizar el rol del antropólogo en este contexto, tal y como sugiere Uesugi, pues, en ocasiones, es precisamente su trabajo el que pone en evidencia esta problemática en el campo y, por tanto, juega un rol relevante en su propio objeto de estudio. 
Finalmente, el artículo de Sarlingo compara la situación de la región argentina de Olavarría con el creciente fenómeno de los monocultivos extensivos de soja en el país y en Latinoamérica en general, y advierte del uso intensivo de agrotóxicos de dudosos efectos para el futuro de la región.

Tal y como sugiere Sarlingo al final de su artículo, la alimentación es también uno de los elementos clave para entender y analizar los riesgos medioambientales actuales. De hecho, este monográfico presenta un bloque de artículos relacionados con esta temática. La alimentación es, de hecho, una de las piezas fundamentales en la construcción del cuerpo contemporáneo, pues el alimento traspasa la barrera entre el mundo y el individuo, asimilando al comensal con aquello que come (Fischler, 1995). En este sentido, la alimentación hace al individuo permeable al mundo externo y es precisamente en este engranaje donde se vuelve especialmente relevante su análisis en este monográfico.

El artículo de Patricia Aguirre analiza los «alimentos funcionales», que son aquellos que han sido diseñados en laboratorio para añadirles sustancias químicas presuntamente beneficiosas para la salud humana. El análisis de estos alimentos supone desgranar las relaciones entre instituciones públicas reguladoras, industrias productoras, instituciones de investigación bioquímica y el mercado de consumo. La autora, reconociendo el fuerte nexo entre salud, patologías y alimentación, recorre la relación entre corporalidades, tipos de ingesta y comensalidad en distintas clases sociales en el contexto sociocultural argentino para relacionarlas con los alimentos funcionales. Según demuestra este artículo, los alimentos funcionales, promocionados como cuasimedicamentos, cuando en realidad contienen abundantes sustancias químicas sintéticas de dudoso efecto beneficioso sobre la salud del comensal, suponen una preeminencia de la corporalidad puramente biológica, dejando de lado los procesos sociales y políticos que conforman las corporalidades en los contextos sociohistóricos concretos. Además, su producción, desarrollo y comercialización ponen en evidencia y reproducen desigualdades sociales en salud de la población a la que se dirigen.

El artículo de Larrea-Killinger y colaboradores examina también el tema de las sustancias químicas en los alimentos, esta vez en un estudio realizado con mujeres embarazadas y lactantes en España, que indaga sobre sus percepciones en torno a los compuestos químicos sintéticos en la alimentación y los riesgos que estos suponen para ellas y sus hijos. El artículo traza las formas en que las mujeres acceden, o no, a la información sobre compuestos químicos en la alimentación, centrando la atención en los consejos que reciben de los expertos sanitarios, por un lado, y de 
sus entornos sociales próximos, por el otro. Dado que la información que reciben es escasa y poco homogénea, el artículo trata de dilucidar las percepciones de estas mujeres sobre la alimentación y los compuestos químicos: qué se considera peligroso y por qué y qué percepciones tienen sobre los procesos de acumulación y transmisión corporal de estas sustancias.

Una vez más, aparece el tema de la escasez de información alrededor de los compuestos potencialmente tóxicos y cómo ese vacío afecta a las percepciones y discursos sociales sobre las corporalidades asociadas a los mismos. En este caso, nos encontramos con una situación en que las mujeres embarazadas y lactantes apenas reciben información por parte de los expertos/as sobre los posibles riesgos derivados de las sustancias químicas y, por tanto, sus percepciones al respecto son heterogéneas, contradictorias e incluso, a veces, confusas. Lejos de los mecanismos de culpabilización que usualmente se aplican sobre las mujeres embarazadas (Lupton, 1999b), en este caso la corporalidad tóxica está invisibilizada en la relación médico-paciente, y esto genera en las mujeres una sensación de aguda indefensión. De nuevo surge el binomio incertidumbre-indefensión, que también se presenta en los artículos de Uesugi, Little y Sarlingo. Es interesante notar que los artículos sobre alimentación, al contrario que estos últimos, no trabajan con poblaciones subalternas o en condiciones políticoeconómicas hostiles, y, aun así, el silenciamiento y la incertidumbre en torno a las sustancias tóxicas deviene una variable social que se mantiene a lo largo de distintas circunstancias y sociedades.

Además, el artículo de Larrea-Killinger y colaboradores aporta una dimensión de género que es interesante para reflexionar sobre las corporalidades tóxicas. Su estudio se centra en mujeres, que por su mayor concentración de células grasas que los hombres, son más proclives a la bioacumulación de sustancias químicas tóxicas en su organismo (Valls-Llobet, 2010), y en un momento vital —embarazo o lactancia- en que estas sustancias pueden ser traspasadas a sus hijos, creando así una cadena generacional de transmisión de tóxicos.

Para cerrar el bloque sobre alimentación, el artículo de Elena Espeitx y Juanjo Cáceres se basa en un estudio sobre las formas de clasificación de alimentos en la sociedad española y los desplazamientos de determinados alimentos dentro de dichas clasificaciones. Este texto enlaza con el de Larrea-Killinger y colaboradores en el sentido de que ambos tratan con las percepciones de la población sobre los alimentos y, en último término, sobre sus potenciales peligros. Es interesante constatar en este estudio, por un lado, que la salud tiene un peso muy importante en la manera en que 
clasificamos los alimentos, y que este concepto está relacionado con la «naturalidad» de los alimentos, aunque dicha «naturalidad» sea definida de formas ambiguas. Así mismo, existe un desplazamiento entre los alimentos pensados como «artificiales» $\mathrm{o}$ «industriales» hacia los alimentos considerados «peligrosos» que, en este sentido, enlaza con los hallazgos de Larrea-Killinger y colaboradores y también con numerosos estudios sobre alimentación (Begueria, 2016; Contreras y Gracia Arnaiz, 2005; Guidonet, 2010). Sin embargo, la peligrosidad de los alimentos no parece ser especialmente relevante para los participantes de este estudio. Además, el contexto social de consumo y la familiaridad con un alimento es un elemento de clasificación alimentaria tan importante como la salud. En este sentido, es interesante observar que las percepciones sobre riesgo alimentario, o la falta de él, y sus correspondientes corporalidades, son socialmente construidas por elementos que no tienen que ver solamente con el alimento en sí, sino también con las formas de consumo y su organización social.

Aunque los artículos del bloque de alimentación son dispares, como ya se ha dicho, coinciden en sus resultados en la poca conciencia social sobre los riesgos alimentarios actuales de origen químico artificial. Es significativo observar que, en sociedades como la española o la argentina, a pesar de que existen numerosos discursos sociales sobre alimentación y riesgo, otros elementos — azúcar o grasas, por ejemplo- sobresalen como problemáticas más acuciantes que la toxicidad de tipo químico.

Los artículos de este monográfico recorren un sendero que traza los contextos sociales, políticos y económicos en que se generan situaciones de silenciamiento o invisibilización de determinadas situaciones o sustancias tóxicas, y describen cómo esa ocultación se traduce en formas de conocimiento - o desconocimiento- de los riesgos medioambientales a los que se exponen distintas poblaciones. A través del análisis de percepciones y conocimientos situados, los autores de este monográfico examinan las corporalidades que emergen en las situaciones particulares descritas en cada uno de los artículos. Estas corporalidades permeables, radicalmente enraizadas en el medio ambiente que las rodea, se encuentran invisibilizadas, silenciadas y, en algunos casos, encubiertas. En este sentido, desde este monográfico proponemos la necesidad de más investigación en este campo desde las distintas ciencias sociales, y especialmente desde la antropología iberoamericana, para exponer, articular y debatir estas corporalidades y su inapelable relación con el mundo en el que vivimos. 


\section{Referencias}

Altman, R.G.; Morello-Frosch, R.; Brody, J.G.; Rudel, R.; Brown, P. y Averick, M. (2008). Pollution comes home and gets personal: women's experience of household chemical exposure. Journal of health and social behavior, 49(4): 417-435.

Apfelbaum, M. (1998). Risques et peurs alimentaires. Paris: Editions Odile Jacob.

Auyero, J. y Swistun, D. (2008). The Social Production of Toxic Uncertainty. American Sociological Review, 73(3): 357-379.

Beck, U. (1986). Risikogesellschaft. Auf dem Weg in eine andere Moderne. Fracfort: Suhrkamp.

Begueria, A. (2016). Un equilibrio imperfecto. Alimentación ecológica, cuerpo y toxicidad. Barcelona: Editorial UOC.

Begueria, A.; Larrea, C.; Muñoz, A.; Zafra, E.; Mascaró-Pons, J. y Porta, M. (2014). Social discourse concerning pollution and contamination in Spain: Analysis of online comments by digital press readers. Contributions to Science, 10: 35-47.

Boudia, S. y Jas, N. (2014). Powerless science? : science and politics in a toxic world. New York : Berghahn.

Bredahl, L. (1999). Consumers Cognitions with Regard to Genetically Modified Foods. Results of a Qualitative Study in Four Countries. Appetite, 33(3): 343-360.

Le Breton, D. (2002). Antropología del cuerpo y modernidad. Buenos Aires: Nueva Visión.

Brown, P.; Zavestoski, S.M.; McCormick, S.; Mandelbaum, J. y Luebke, T. (2001). Print media coverage of environmental causation of breast cancer. Sociology of Health and Illness, 23(6): 747-775.

Butz, P.; Needs, E.C.; Baron, A.; Bayer, O.; Geisel, B.; Gupta, B.; Oltersdorf, U. et al. (2003). Consumer attitudes to high pressure food processing. Food, Agriculture \& Environment, 1(1).

Carson, R. (1964). Primavera silenciosa. Barcelona: Caralt.

Checker, M. (2007). “But I Know It's True”: Environmental Risk Assessment, Justice, and Anthropology. Human Organization, 66(2): 112-124.

Contreras, J. y Gracia Arnaiz, M. (2005). Alimentación y cultura: perspectivas antropológicas. Barcelona: Ariel.

Douglas, M. (1994). Risk and blame: essays in cultural theory. London: Routledge.

Douglas, M. y Wildavsky, A.B. (1982). Risk and culture: an essay on the selection of technological and environmental dangers. Berkeley: University of California Press.

Elias, N. (1989). El Proceso de la civilización: investigaciones sociogenéticas y psicogenéticas. México: Fondo de Cultura Económica.

Erickson, B.E. (2007). Toxin or Medicine? Explanatory Models of Radon in Montana Health Mines. Medical Anthropology Quarterly, 21(1): 1-21.

Espeitx, E. y Cáceres, J. (2012). Les noves tecnologies aplicades a l'alimentació: factors d'acceptació i rebuig a Catalunya. Barcelona.

Fischler, C. (1995). El (H)omnívoro: el gusto, la cocina y el cuerpo. Barcelona: Anagrama. Fischler, C. (2002). Food Selection and Risk Perception. En Food selection. From genes to culture. H. Anderson, J. Blundell y M. Chiva, Eds. Levallois-Perret: Danone Institute.

Foucault, M. (2009). Vigilar y castigar: nacimiento de la prisión. México: Siglo Veintiuno. 
Goldstein, D.M. (2017). Invisible harm: science, subjectivity and the things we cannot see. Culture, Theory and Critique, 58(4): 321-329.

Guidonet, A. (2010). ¿Miedo a comer? Crisis alimentaria en contextos de abundancia. Barcelona: Icaria.

Haenn, N. y Wilk, R.R. (2006). The environment in anthropology: a reader in ecology, culture, and sustainable living. London: New York University Press.

Harthorn, B.H. (2003). Safe Exposure? Perceptions of Health Risks from Agricultural Chemicals among California Farmworkers. Risk, culture, and health inequality: shifting perceptions of danger and blame.

Hunt, L.; Tinoco, R.; Schwartz, N. y Halperin, D. (1999). Balancing Risk and Resources: Applying Pesticides without Using Protective Equipment in Southern Mexico. Anthropology in public health: bridging differences in culture and society.

Jensen, M. y Blok, A. (2008). Pesticides in the Risk Society: The View from Everyday Life. Current Sociology, 56(5): 757-778.

Kavanagh, A.M. y Broom, D.H. (1998). Embodied risk: my body, myself? Social science \& medicine, 46(3): 437-444.

Kleinman, A. (1980). Patients and healers in the context of culture: an exploration of the borderland between anthropology, medicine, and psychiatry. Berkeley: University of California Press.

Larrea-Killinger, C.; Muñoz, A. y Mascaró, J. (2017). Cuerpos tóxicos: la percepción del riesgo de la contaminación interna por compuestos químicos en España. Salud Colectiva, 13(2): 225-237.

Larrea-Killinger, C.; Muñoz, A.; Mascaró, J.; Zafra, E. y Porta, M. (2017). Discourses on the Toxic Effects of Internal Chemical Contamination in Catalonia, Spain. Medical Anthropology, 36(2): 125-140.

Latouche, K.; Rainelli, P. y Vermersch, D. (1998). Food safety issues and the BSE scare: Some lessons from the French case. Food Policy, 23(5): 347-356.

Latour, B. (2004). How to Talk About the Body? The Normative Dimension of Science Studies. Body \& Society, 10(2-3): 205-229.

Lora-Wainwright, A. (2013). Plural forms of evidence and uncertainty in environmental health: A comparison of two Chinese cases. Evidence and Policy, 9(1): 49-64.

Lupton, D. (1999a). Risk. London; New York: Routledge.

Lupton, D. (1999b). Risk and the ontology of pregnant embodiment. En Risk and sociocultural theory: new directions and perspectives. Cambridge: Cambridge University.

Lupton, D. (2013). Risk and emotion: towards an alternative theoretical perspective. Health, Risk \& Society, 1-14.

Mascaró Pons, J. (2013). Una proposta d'anàlisi de l'imaginari cultural del cos i de la corporalitat tòxica. Quaderns de l'Institut Català d'Antropologia, 18(2): 145-155.

Muñoz, A.; Larrea, C.; Zafra, E. y Begueria, A. (2014). Las responsabilidades sobre las sustancias químicas y los Compuestos Tóxicos Persistentes (CTP): una perspectiva antropológica sobre los riesgos. Periferias, Fronteras y Diálogos: 4386-4406.

Murphy, M. (2008). Chemical Regimes of Living. Source: Environmental History, 13(4): 695-703. 
Murphy, M. (2017a). Alterlife and Decolonial Chemical Relations. Cultural Anthropology, 32(4): 494-503.

Murphy, M. (2017b). What Can't a Body Do? Catalyst: Feminism, Theory, Technoscience, 3(1).

Palis, F.G.; Flor, R.J.; Warburton, H. y Hossain, M. (2006). Our farmers at risk: Behaviour and belief system in pesticide safety. Journal of Public Health, 28(1): 43-48.

Petryna, A. (2013). Life exposed: biological citizens after Chernobyl. Princeton University Press.

Phillips, T. (2010). Debating the legitimacy of a contested environmental illness: A case study of multiple chemical sensitivities (MCS). Sociology of Health and Illness, 32(7): 1026-1040.

Porta, M.; Puigdomènech, E. y Ballester Díez, F. (2009). Nuestra contaminación interna: concentraciones de compuestos tóxicos persistentes en la población española. Madrid: Libros de la Catarata.

Quandt, S.A.; Arcury, T.A; Austin, C.K. y Saavedra, R.M. (1998). Farmworker and Farmer Perceptions of Farmworker Agricultural Chemical Exposure in North Carolina. Human Organization.

Scheper-Hughes, N. y Lock, M.M. (1987). The mindful body: a prolegomenon to future work in medical anthropology. Medical anthropology quarterly, 1(1): 6-41.

Shapiro, N. y Kirksey, E. (2017). Chemo-Ethnography: An Introduction. Cultural Anthropology, 32(4): 481-493.

Sicotte, D. (2009). Power, profit and pollution: The persistence of environmental injustice in a company town. Human Ecology Review, 16(2): 141-150.

Singer, M. (2011). Down Cancer Alley: The Lived Experience of Health and Environmental Suffering in Louisiana's Chemical Corridor. Medical Anthropology Quarterly, 25(2): 141-163.

Snipes, S.A.; Thompson, B.; O’Connor, K.; Shell-Duncan, B.; King, D.; Herrera, A.P. y Navarro, B. (2009). "Pesticides protect the fruit, but not the people": using communitybased ethnography to understand farmworker pesticide-exposure risks. American journal of public health, 99(3).

Soares de Freitas, M.C., de Souza Minayo, M.C., Lopes Pena, P.G. y Miranda dos Santos, N.M. (2012). Un ambiente enfermo: significados de la contaminación industrial en Isla de Maré, Bahía, Brasil. Desacatos, 39: 73-88.

Tulloch, J. y Lupton, D. (2003). Risk and everyday life. London: Sage.

Valls-Llobet, C. (2010). Contaminación ambiental y salud de las mujeres. Investigaciones Feministas, 1(0): 149-159.

Vigarello, G. (1991). Lo Limpio y lo sucio: la higiene del cuerpo desde la Edad Media. Madrid: Alianza.

Wynne, B. (1996). May the sheep safely graze? A reflexive view of the expert-lay knowledge divide. Risk, Environment and Modernity: Towards a New Ecology: 44-83.

Zafra Aparici, E.; Muñoz García, A. y Larrea-Killinger, C. (2016). ¿Sabemos lo que comemos?: Percepciones sobre el riesgo alimentario en Cataluña, España. Salud Colectiva, 12(4): 505. 
